\title{
Systems Biology of Halophilic Archaea
}

\author{
Dieter Oesterhelt \\ Max Planck Institute of Biochemistry, \\ Department of Membrane Biochemistry, \\ Martinsried, Germany \\ oesterhe@biochem.mpg.de
}

\begin{abstract}
Systems biology is spread over all branches of life science and attracts biologists, mathematicians, physicists, computer scientists, and engineers equally. Full of promises and visions it often signalizes that the in silico eucaryotic cell is close to realization and experimental work will be needed in the future only for confirmation. At this point science becomes fiction and destroys the great potential of interdisciplinary research aiming for added value in describing a living system or its composing modules by theoretical/simulation approaches on the basis of experimental facts. As a reliable working definition of molecular systems biology the following is useful: Modelling of cells or a modules of cells with an incomplete data set. The model (ensemble of models) must have predictive value to induce experiments which lead to falsification (verification) of subsets of models until, on the basis of available data, optimally only one model is left. The approach can be either "bottom up" or "top down". We use halophilic archaea, especially the model organism Halobacterium salinarum for systems biological experiments. These procaryotes living in concentrated brines offer biochemical features which make them very suitable for systematic analysis. A first module is signal transduction where photon absorption via two photoreceptors causes three different reactions of the target, which is the flagellar motor. The system guarantees a balanced response of the cell to light for active search of the optimal conditions for photosynthesis. Experimentally, quantitative data can be collected, which link the size of stimulus to the reaction time of the flagellar motor. Further, genome wide data on members of the network, their molecular properties and protein protein interactions were made available. Altogether a model was developed, which allows to simulate all experimental results reported so far. Bioenergetics are a second module, which is ready for modelling with a bottom up approach and the central metabolism of the cell presents an example of top down modelling with about 800 reactions in the cell. Experimental data on the course of sixteen amino acids added to the growth medium as carbon source and on the rate growth were collected and a model created which is able to quantitatively predict growth curve and carbon source usage. The lecture will give account on the details of the experimental methods used, describe the modelling approaches and summarize the results, we so far obtained.
\end{abstract}

